Published in final edited form as:

Ann Surg Oncol. 2018 September ; 25(9): 2587-2595. doi:10.1245/s10434-018-6496-4.

\title{
A National Study of the Use of Asymptomatic Systemic Imaging for Surveillance Following Breast Cancer Treatment
}

\author{
Jessica R. Schumacher, PhD ${ }^{1}$, Heather B. Neuman, MD, MS ${ }^{1}$, George J. Chang, MD, MS ${ }^{2}$, \\ Benjamin D. Kozower, MD, MPH ${ }^{3}$, Stephen Edge, $\mathbf{M D}^{4}$, Menggang $\mathrm{Yu}, \mathrm{PhD}^{5}$, David J \\ Vanness, PhD $^{6}$, Yajuan $\mathrm{Si}$, PhD $^{5,6}$, Elizabeth A. Jacobs, $\mathbf{M D}^{7}$, Amanda B. Francescatti, $\mathbf{M S}^{8}$, \\ Patricia Spears ${ }^{9}$, Jeffrey Havlena, MS ${ }^{1}$, Taiwo Adesoye, MD, MPH ${ }^{1}$, Daniel McKellar, MD ${ }^{8}$, \\ David Winchester, MD $^{8}$, Elizabeth Burnside, MD, MPH, MS ${ }^{10}$, Caprice C. Greenberg, MD, \\ MPH $^{1}$, and Alliance ACS-CRP CCDR Breast Cancer Surveillance Working Group \\ 1.Wisconsin Surgical Outcomes Research Program, Department of Surgery, University of \\ Wisconsin-Madison School of Medicine and Public Health, Madison, WI \\ 2. Department of Surgical Oncology and Department of Health Services Research, The University \\ of Texas MD Anderson Cancer Center, Houston, TX \\ 3.Department of Surgery, Washington University School of Medicine in St. Louis, St. Louis, MO \\ 4. Roswell Park Cancer Institute, Buffalo, NY \\ 5.Department of Biostatistics \& Medical Informatics, University of Wisconsin-Madison, Madison, \\ WI \\ 6. Department of Population Health Sciences, University of Wisconsin-Madison School of Medicine \\ and Public Health, Madison, WI \\ 7.Department of Medicine, University of Wisconsin-Madison School of Medicine and Public \\ Health, Madison, WI \\ 8.American College of Surgeons, Commission on Cancer, Chicago, IL \\ 9.Department of Population Health and Pathobiology, North Carolina State University, Raleigh, NC \\ 10. Department of Radiology, University of Wisconsin-Madison
}

\begin{abstract}
Background: Though not guideline recommended, studies suggest $50 \%$ of locoregional breast cancer patients undergo systemic imaging during follow-up, prompting its inclusion as a Choosing Wisely measure of potential overuse. Most studies rely on administrative data that cannot delineate
\end{abstract}

Corresponding Author: Caprice Greenberg, MD MPH, Professor, Department of Surgery, Director, Wisconsin Surgical Outcomes Research Program (WiSOR), University of Wisconsin School of Medicine and Public Health, Phone: 608.263.7309| greenberg@surgery.wisc.edu.

Disclaimer: The American College of Surgeons and the Commission on Cancer have not verified and are not responsible for the analytic or statistical methodology employed, or the conclusions drawn from these data by the investigator. Further, the contents of this publication are solely the responsibility of the authors and do not necessarily represent the views of NIH or the Patient-Centered Outcomes Research Institute, its Board of Governors or Methodology Committee.

Conflicts of Interest: None 
scan intent (prompted by signs/symptoms versus asymptomatic surveillance). This is a critical gap, as intent is the only way to distinguish overuse from appropriate care. Our objective was to assess surveillance systemic imaging post-breast cancer treatment in a national sample accounting for scan intent.

Methods: A stage-stratified random sample of 10 women with stage II-III breast cancer in 20062007 was selected from each of 1,217 Commission on Cancer accredited facilities for a total of 10,838 patients. Registrars abstracted scan type (CT, non-breast MRI, bone scan, PET/CT) and intent (cancer-related versus not, asymptomatic surveillance versus not) from medical records for 5 years post-diagnosis. Data was merged with each patient's corresponding National Cancer Database record, containing sociodemographic and tumor/treatment information.

Results: Of 10,838 women, $30 \%$ had $\geq 1$ and $12 \%$ had $\geq 2$ systemic surveillance scans during a four-year follow-up period. Patients were more likely to receive surveillance imaging in the first follow-up year (lower proportions during subsequent years), and if they had ER/PR negative tumors.

Conclusions: Locoregional breast cancer patients undergo asymptomatic systemic imaging during follow-up despite guidelines recommending against it, but at lower rates than previously reported. Providers appear to use factors that confer increased recurrence risk to tailor decisions about systemic surveillance imaging, perhaps reflecting limitations of data on which current guidelines are based.

\section{Keywords}

Breast Cancer; Systemic Imaging; Cancer Survivorship; Health Services Research

\section{INTRODUCTION}

Improvements in early detection and treatment of locoregional breast cancer have increased the 5-year survival rate to $90 \%$. Over 3 million breast cancer survivors receive follow-up care in the U.S. ${ }^{1-3}$ Clinical guidelines published by the American Society of Clinical Oncology (ASCO) and National Comprehensive Cancer Network recommend frequent clinical follow-up visits to detect new primary cancers, recurrence, and treatment side effects. However, guidelines specifically recommend against asymptomatic systemic imaging for surveillance of metastatic disease, with systemic imaging only recommended in the presence of suspicious signs or symptoms. ${ }^{4-6}$

Despite clinical practice guidelines, population-level studies suggest significant overuse of systemic imaging following breast cancer treatment. In these studies, nearly half of all patients with locoregional breast cancer undergo at least one advanced imaging study (CT scan, MRI, bone scan, PET scan) to detect distant recurrence following breast cancer treatment. ${ }^{7-10}$ This prompted ASCO to partner with the American Board of Internal Medicine Foundation Choosing Wisely campaign to add asymptomatic systemic imaging of breast cancer survivors as an overuse measure, and as a potential target for improving care and reducing cost. ${ }^{11,12}$ 
Population-level studies to date, however, have primarily relied on administrative data that are unable to differentiate between imaging prompted by a patient symptom or clinical sign, and asymptomatic surveillance. Without such information, it is impossible to distinguish true overuse from guideline-concordant imaging due to signs or symptoms. Consequently, claims-based studies may over-estimate systemic imaging use for routine surveillance. Furthermore, administrative data is unable to evaluate whether tumor characteristics, and therefore recurrence risk, are driving observed utilization patterns. We sought to address these gaps using abstracted medical records from a national sample of women with breast cancer diagnosed at 1,217 facilities.

\section{METHODS}

\section{Setting and Population}

This study used a national cancer registry augmented with primary data collection. The National Cancer Database (NCDB), ${ }^{13,14}$ a joint program of the American College of Surgeons, Commission on Cancer (CoC), and the American Cancer Society, captures 70\% of newly diagnosed cancers in the U.S. ${ }^{13,15}$ To maintain accreditation and ensure data quality, facilities are required to follow $90 \%$ of patients from diagnosis until death, regardless of where patients receive follow-up care. Using a standardized abstraction manual, trained registrars collect information on sociodemographic, tumor, and treatment factors. ${ }^{16}$ Imaging and recurrence were abstracted to supplement standard elements as part of a special study initiative. Only de-identified data was provided to investigators and therefore the study was deemed exempt as non-human subjects research by the University of Wisconsin IRB.

\section{Eligibility Criteria and Sampling Strategy}

Women over age 18 were eligible if they had a first diagnosis of stage II-III breast cancer (ICDO-3 code:C50.0-C50.9) in 2006-2007. These years were selected to allow 5 years of follow-up through 2013, the most recent data available. Such patients, who were treated with curative intent (including surgery), were considered most likely to undergo systemic imaging because they are at higher risk of recurrence than women diagnosed at earlier stages (0-I).

A stage-stratified random sample of 10 patients ( 7 stage II, 3 stage III) was selected from all $1,231$ facilities accredited by the $\mathrm{CoC}$ in 2006-2007 ( $\mathrm{n}=11,478)$. We sought to maximize generalizability by randomly selecting a small number of patients at all $\mathrm{CoC}$ institutions. The sampling strategy was designed to achieve a similar ratio of stage II to stage III patients as observed nationally (for census of patients meeting inclusion criteria, $73.9 \%$ were stage II, and 26.1\% were stage III in NCDB), while minimizing abstraction burden. 1,217 facilities participated (99\%), with 11,360 patient records submitted. All but 17 facilities diagnosed and treated at least one stage III patient.

Trained facility registrars solicited and reviewed relevant patient records from their own and outside facilities beginning at the end of active treatment (approximated by 10 months postdiagnosis) and continued until: 1) the end of the fifth year post-diagnosis, 2) distant recurrence, or 3) death, whichever was first. Follow-up information, including systemic 
imaging (CT, MRI, bone scan, PET) was abstracted using a standardized abstraction manual and entered via secure web platform. The type and indication of each imaging study was recorded, along with date performed, and results. Her2neu status, not abstracted routinely in 2006-2007, was also collected. Key fields (e.g., date of death) were confirmed. Registrars abstracted recurrence because this element is unreliable and missing for $20 \%$ of patients. ${ }^{17}$ Recorded data elements were merged with a patient's corresponding record in the NCDB.

Patients were excluded if they had delayed surgery ( $>1$ year from diagnosis, $\mathrm{n}=17$ ) or had recurrence, new primary cancer, death, or were lost to follow-up before the start of the surveillance period (10 months from diagnosis) $(\mathrm{n}=505)$.

\section{Surveillance Imaging}

Systemic scans were grouped into 3 categories according to the area imaged: bone scans, body imaging (non-breast MRI, CT, and/or PET of the chest, abdomen, and/or pelvis), and brain imaging (CT and MRI). Imaging was considered surveillance if classified as "surveillance imaging in the absence of new signs or symptoms (asymptomatic)." Registrars were guided to high yield locations in the medical record to obtain this information (primary: radiology/imaging reports from their own/other institutions; secondary: notes from clinic and consult visits from primary care, oncologist, or other providers, paying particular attention to references to symptoms or instructions to schedule scans within a specified time period). Often, multiple data sources were combined to evaluate a given imaging study. In order to compare to previous studies, a composite variable_-"cancer-related scans"included surveillance scans and scans with the following clinical indications: follow-up for new sign/symptom; follow-up for suspicious finding on other imaging; imaging performed as part of staging work-up for newly detected malignancy (new primary or recurrence).

\section{Sociodemographic and Cancer-Related Characteristics}

Sociodemographic characteristics included age, race, Hispanic ethnicity, zip code, level of education, insurance status, and rural/urban residence. Charlson/Deyo score, categorized as 0 or $1+$ reflecting the general health of breast cancer patients, was also available. Tumorrelated factors included AJCC pathologic stage, grade, and histology. Estrogen receptor (ER), progesterone receptor (PR), and Her2neu status were combined to create four molecular subtype groups (ER or PR+/Her2neu-; ER and PR-/Her2neu-; ER or PR+/ Her2neu+; ER and PR-/Her2neu+). ${ }^{18}$ Treatment included surgery type and receipt of radiation and/or chemotherapy. Facility type was also included.

\section{Quality Assurance}

Pilot Study: Full study information was abstracted for 180 patients at $18 \mathrm{CoC}$-accredited facilities meeting inclusion criteria (10 patients per facility), confirming feasible and valid abstraction.

Planned Reliability Study: A random 5\% sample of patients who received their diagnosis and/or first course treatment at multiple facilities $(n=537)$ was selected. Registrars at the second outside facility were asked to abstract identical information for the same patient to assess abstraction reliability. This was possible because registrars at $\mathrm{CoC}$ facilities 
are required to track patients who receive follow-up care at multiple facilities as a part of accreditation. ${ }^{13}$ Most cancer-related care was received at the primary facility, where registrars had direct access to more complete follow-up information through that facility's medical records. In contrast, the registrar at the second facility had to request a greater number of records. A total of 1,240 scans of the same type were abstracted. Of these, 418 were advanced imaging scans. The observed percent agreement for surveillance versus symptom/follow-up advanced imaging was $79.4 \%$ (expected $=51.0 \%$ ), while the kappa was $0.6(\mathrm{Z}=11.9, \mathrm{p}<0.001)$. This represents a lower-limit reliability estimate, since by definition primary facilities had direct access to more complete medical information.

\section{Statistical Analysis}

The proportion of patients receiving any cancer-related and asymptomatic surveillance systemic imaging was calculated. The broadest definition of surveillance included patients receiving $\geq 1$ scans for asymptomatic surveillance during follow-up, while a more conservative definition required $\geq 2$ of the same scan type (head, bone, body). Among women who underwent $\geq 1$ surveillance imaging scans during the follow-up period, frequencies of the imaging types performed were estimated.

The percent who received $\geq 1$ surveillance systemic imaging scans was compared by molecular subtype group within each of four follow-up years, beginning at the start of the surveillance period (Year 1:10-24 months; Year 2:24-36 months; Year 3:36-48 months; Year 4:48-60 months). Patients were censored the year after distant recurrence, death, or when they were lost to follow-up.

Using multivariable logistic regression, we assessed factors associated with the receipt of $\geq 1$ asymptomatic surveillance systemic imaging scans during follow-up. A missingness indicator variable was included for measures with missing information. A second model was fit predicting receipt of $\geq 2$ surveillance scans. Model findings are not reported, as they were consistent with models predicting $\geq 1$ surveillance scans. All models were estimated with robust standard errors. Adjusted average predicted probabilities were estimated for all factors to facilitate interpretation. ${ }^{19}$ Analyses were conducted using Stata v13.

\section{RESULTS}

Of 10,838 women, $72 \%$ were under age $70,41 \%$ had government-provided insurance, $71 \%$ were ER or PR+, and 19\% were HER2neu+ (Table 1).

Forty-eight percent $(\mathrm{n}=5,220)$ of women received $\geq 1$ cancer-related advanced imaging scans during follow-up (Table 2). Once intent of scan was considered, $30 \%$ of the sample $(\mathrm{n}=3,254)$ received $\geq 1$ asymptomatic surveillance systemic imaging scans, while $12 \%$ $(\mathrm{n}=1,308)$ had $\geq 2$ surveillance scans of the same type (body, brain, or bone). Of women who received $\geq 1$ systemic imaging scans for surveillance, the majority received body imaging and/or bone scans (Figure 1).

The proportion of patients who received $\geq 1$ surveillance systemic imaging scans was highest during the first follow-up year, with lower proportions during subsequent years (Table 3).

Ann Surg Oncol. Author manuscript; available in PMC 2019 September 01. 
During year $1,13.5 \%$ of patients received an asymptomatic systemic imaging study, $10.0 \%$ in year $2,8.1 \%$ in year 3 , and $6.0 \%$ in year 4 . The proportion of patients receiving asymptomatic systemic imaging in year 1 was lowest for patients with ER or PR+/Her2neutumors (12.4\%) and highest for patients with ER and PR-/Her2neu+ tumors (19.2\%).

Tumor and treatment, but not the majority of sociodemographic or comorbidity factors, were significantly related to the receipt of $\geq 1$ asymptomatic systemic imaging scans for surveillance during follow-up (Table 4). One exception was that patients living in urban areas had greater odds of receiving $\geq 1$ surveillance scans than patients in rural areas $(\mathrm{OR}=1.51,95 \% \mathrm{CI}=1.12-2.04)$. Tumor-related factors associated with asymptomatic systemic surveillance imaging included pathologic stage and molecular subtype. Specifically, patients diagnosed with stage III as compared to stage II disease had higher odds of asymptomatic advanced imaging for surveillance ( $\mathrm{OR}=1.46,95 \% \mathrm{CI}=1.32-1.62)$. In addition, patients with ER and PR-/Her2neu- tumors ( $\mathrm{OR}=1.14,95 \% \mathrm{CI}=1.00-1.30)$, ER or $\mathrm{PR}+/$ Her2neu+ tumors $(\mathrm{OR}=1.18,95 \% \mathrm{CI}=1.04-1.35)$, and ER and PR-/Her2neu+ $(\mathrm{OR}=1.17,95 \% \mathrm{CI}=0.99-1.38)$ tumors had higher odds of receiving an asymptomatic systemic imaging for surveillance than patients diagnosed with ER or PR+/Her2neu- tumors. Receipt of chemotherapy $(\mathrm{OR}=1.92,95 \% \mathrm{CI}=1.68-2.20)$ and mastectomy with $(\mathrm{OR}=1.42$, $95 \% \mathrm{CI}=1.27-1.60)$ or without $(\mathrm{OR}=1.21,95 \% \mathrm{CI}=1.08-1.35)$ radiation were also significantly associated with greater odds of receiving asymptomatic systemic imaging.

\section{DISCUSSION}

The current growth of U.S. healthcare expenditures is not sustainable. One attempt to control costs relies on identifying and decreasing unproven or otherwise not recommended care. For breast cancer, the use of surveillance systemic imaging for asymptomatic individuals following breast cancer treatment is considered a high value target by ASCO and the Choosing Wisely campaign. ${ }^{11,12}$

Consistent with prior administrative claims-based studies, ${ }^{7-10}$ we found that approximately $50 \%$ of women with stage II-III breast cancer had $\geq 1$ systemic imaging scans for a cancerrelated indication during a five-year follow-up period starting at diagnosis. However, when intent of the scan was considered, that percentage dropped; $30 \%$ of patients in our study underwent at least one systemic imaging scan in the absence of symptoms. This is consistent with the one previous study that was able to account for intent of systemic imaging. ${ }^{20} \mathrm{We}$ also found that only $12 \%$ of patients received $\geq 2$ asymptomatic scans of the same type during the study period. If you consider true use of surveillance imaging to be the longitudinal monitoring with asymptomatic imaging over time, our data suggest that a smaller proportion of women have regular or annual advanced imaging screening for metastases than was anticipated.

Perhaps more importantly, we demonstrate that the use of systemic imaging in the absence of symptoms is primarily driven by tumor and treatment factors associated with increased risk of distant recurrence. Although breast cancer treatments are increasingly tailored to patient risk factors (e.g., molecular subtype), current follow-up guidelines and recommendations, including imaging, are not personalized. According to a growing body of 
evidence, risk and timing of recurrence varies, with risk being highest in the first 5 years for patients with ER/PR negative tumors. ${ }^{18,21}$ In our study, ER/PR- patients were more likely than ER/PR+ patients to have asymptomatic imaging for surveillance (regardless of Her2neu status), suggesting recurrence risk may play a role in clinicians' decision making regarding systemic imaging for asymptomatic surveillance. Findings further suggest that patients were more likely to receive advanced imaging earlier in the course of follow-up, especially during the first year when risk of recurrence and patient anxiety may be highest. ${ }^{18,21}$ Such tailored use of asymptomatic imaging is conceptually consistent with the Choosing Wisely aim to improve quality of care, which includes providing care that is personalized and equitable.. ${ }^{22}$

The tailoring of surveillance may also represent physician uncertainty about the optimal follow-up approach, given limitations around the data supporting current follow-up imaging recommendations. Current guideline recommendations are based on randomized trials showing the detection of distant recurrence by asymptomatic systemic surveillance confers no survival or health-related quality of life advantage relative to less intensive follow-up (provider visits with history/physical examination). ${ }^{23-27}$ However, these older studies do not account for advances in systemic imaging quality and treatment effectiveness, which could result in providers ordering systemic imaging for some asymptomatic patients despite guideline recommendations.

There are several limitations to consider. First, we sampled patients at the facility level using the ratio of stage II-III patients observed nationally. Though this approach allowed for the reliable capture of the breadth of imaging practices in the U.S., it precludes an investigation of provider factors associated with imaging and underrepresents higher volume centers where a larger proportion of patients receive care. In addition, the study was limited to 2006-2007 diagnoses to allow for the collection of 5-year follow-up information. Her2neu status was not recorded consistently. Further, Trastuzumab was not routinely administered for patients with Her2neu+ tumors during these years and, despite reabstraction of this information, it was missing for $7 \%$ of patients.

In spite of these limitations, findings indicate the use of surveillance imaging to detect asymptomatic distant recurrence in women with stage II-III breast cancer is lower than previously believed. Women who do receive imaging tend to be at highest risk of recurrence, suggesting that clinicians are tailoring their recommendations for use of surveillance imaging based on their perceptions of patient's recurrence risk. Future efforts should focus on studying the effectiveness of routine surveillance imaging in subgroups of patients thought to be at increased risk of recurrence, to determine whether the clinical practices observed in our study are warranted. Until such data are available, we should continue efforts to decrease utilization of surveillance systemic imaging.

\section{Acknowledgments:}

An abstract based on these findings was selected for a podium presentation at the 2016 AcademyHealth Annual Research Meeting. Boston, MA: June 2016.

The authors wish to thank the cancer registrars and cancer physician liaisons at all participating Commission on Cancer accredited facilities and National Cancer Database staff for their contributions and dedication to this project. Other members of the Alliance American College of Surgeons Clinical Research Program (ACS-CRP) Cancer Care 
Delivery Research PCORI Breast Cancer Surveillance Working Group include: Karla Ballman, PhD; Deborah Schrag, MD, MPH; Ann Partridge, MD, MPH; Kathryn Ruddy, MD; Patrick Gavin, RPh; Bettye Green, RN; Jane Perlmutter, PhD, MBA; Elizabeth Berger, MD; Rinaa Punglia, MD, MPH; Ronald Chen, MD, MPH; Nicole Brys, MPH; Ying Zhang.

Disclosure of Funding: Research reported in this manuscript was funded through a Patient Centered Outcomes Research Institute (PCORI) Award (CE-1304-6543) and the National Cancer Institute at the National Institutes of Health (NIH) (grant number U10CA180821 to the Alliance for Clinical Trials in Oncology). Further funding came from the Building Interdisciplinary Research Careers in Women's Health Scholar Program and University of Wisconsin Carbone Comprehensive Cancer Center Academic Oncologist Training Program (Neuman, NIH K12 HD055894, 5K12CA087718), Clinical Translational Science Award (Ruddy, UL1 TR000135, KL2TR000136-09) from the National Center for Advancing Translational Sciences, a component of the National Institutes of Health $(\mathrm{NIH})$, and the National Cancer Institute funded Surgical Oncology Research Training Program (Adesoye, T32CA090217).

\section{REFERENCES}

1. Howlander N, Noone AM, Krapcho M, et al. SEER Cancer Statistics Review, 1975-2011. Bethesda, MD: National Cancer Institute; 2014.

2. Siegel R, DeSantis C, Virgo K, et al. Cancer treatment and survivorship statistics, 2012. CA Cancer J Clin. 2012;62(4):220-241. [PubMed: 22700443]

3. Siegel R, Naishadham D, Jemal A. Cancer statistics, 2012. CA: a cancer journal for clinicians. 2012;62(1):10-29. [PubMed: 22237781]

4. Khatcheressian JL, Hurley P, Bantug E, et al. Breast cancer follow-up and management after primary treatment: American Society of Clinical Oncology clinical practice guideline update. Journal of clinical oncology : official journal of the American Society of Clinical Oncology. 2013;31(7):961-965. [PubMed: 23129741]

5. Khatcheressian JL, Wolff AC, Smith TJ, et al. American Society of Clinical Oncology 2006 update of the breast cancer follow-up and management guidelines in the adjuvant setting. Journal of clinical oncology : official journal of the American Society of Clinical Oncology. 2006;24(31):5091-5097. [PubMed: 17033037]

6. NCCN Clinical Practice Guidelines in Oncology Version 2.2011. 2011; http://www.nccn.org/ clinical.asp. Accessed 9/20/11.

7. Grunfeld E, Hodgson DC, Del Giudice ME, et al. Population-based longitudinal study of follow-up care for breast cancer survivors. Journal of oncology practice / American Society of Clinical Oncology. 2010;6(4):174-181.

8. Panageas KS, Sima CS, Liberman L, et al. Use of high technology imaging for surveillance of early stage breast cancer. Breast cancer research and treatment. 2012;131(2):663-670. [PubMed: 21947679]

9. Hahn EE, Hays RD, Kahn KL, et al. Use of Imaging and Biomarker Tests for Posttreatment Care of Early-Stage Breast Cancer Survivors. Cancer-Am Cancer Soc. 2013;119(24):4316-4324.

10. Geurts SM, de Vegt F, Siesling S, et al. Pattern of follow-up care and early relapse detection in breast cancer patients. Breast cancer research and treatment. 2012;136(3):859-868. [PubMed: 23117854]

11. Schnipper LE, Smith TJ, Raghavan D, et al. American Society of Clinical Oncology identifies five key opportunities to improve care and reduce costs: the top five list for oncology. Journal of clinical oncology : official journal of the American Society of Clinical Oncology. 2012;30(14): 1715-1724. [PubMed: 22493340]

12. American Board of Internal Medicine Foundation. Choosing Wisely Clinician Lists. http:// www.choosingwisely.org/clinician-lists. Accessed 04/11/2016.

13. Bilimoria KY, Stewart AK, Winchester DP, et al. The National Cancer Data Base: a powerful initiative to improve cancer care in the United States. Annals of surgical oncology. 2008;15(3): 683-690. [PubMed: 18183467]

14. Williams RT, Stewart AK, Winchester DP. Monitoring the delivery of cancer care: Commission on Cancer and National Cancer Data Base. Surgical oncology clinics of North America. 2012;21(3): 377-388, vii. [PubMed: 22583987] 
15. Winchester DP, Stewart AK, Bura C, et al. The National Cancer Data Base: A clinical surveillance and quality improvement tool. J Surg Oncol. 2004;85(1):1-3. [PubMed: 14696080]

16. Commission on Cancer. Facility Oncology Registry Data Standards. Chicago, IL2013.

17. In H, Bilimoria KY, Stewart AK, et al. Cancer recurrence: an important but missing variable in national cancer registries. Annals of surgical oncology. 2014;21(5):1520-1529. [PubMed: 24504926]

18. Cossetti RJ, Tyldesley SK, Speers CH, et al. Comparison of breast cancer recurrence and outcome patterns between patients treated from 1986 to 1992 and from 2004 to 2008. Journal of clinical oncology : official journal of the American Society of Clinical Oncology. 2015;33(1):65-73. [PubMed: 25422485]

19. Williams R Using the margins command to estimate and interpret adjusted predictions and marginal effects. Stata J. 2012;12(2):308-331.

20. Hahn EE, Tang T, Lee JS, et al. Use of posttreatment imaging and biomarkers in survivors of earlystage breast cancer: Inappropriate surveillance or necessary care? Cancer-Am Cancer Soc. 2016;122(6):908-916.

21. Colleoni M, Sun Z, Price KN, et al. Annual Hazard Rates of Recurrence for Breast Cancer During 24 Years of Follow-Up: Results From the International Breast Cancer Study Group Trials I to V. Journal of clinical oncology : official journal of the American Society of Clinical Oncology. 2016;34(9):927-935. [PubMed: 26786933]

22. Institute of Medicine - Committee on Quality of Health Care in America. Crossing the quality chasm: A new health system for the 21 st century. Washington, D.C.: National Academy Press; 2001.

23. Palli D, Russo A, Saieva C, et al. Intensive vs clinical follow-up after treatment of primary breast cancer: 10-year update of a randomized trial. Jama-J Am Med Assoc. 1999;281(17):1586-1586.

24. Ghezzi P, Magnanini S, Rinaldini M, et al. Impact of Follow-up Testing on Survival and HealthRelated Quality-of-Life in Breast-Cancer Patients - a Multicenter Randomized Controlled Trial. Jama-J Am Med Assoc. 1994;271(20):1587-1592.

25. Delturco MR, Palli D, Cariddi A, et al. Intensive Diagnostic Follow-up after Treatment of Primary Breast-Cancer - a Randomized Trial. Jama-J Am Med Assoc. 1994;271(20):1593-1597.

26. Liberati A The GIVIO trial on the impact of follow-up care on survival and quality of life in breast cancer patients. Ann Oncol. 1995;6:41-46.

27. Rojas MP, Telaro E, Russo A, et al. Follow-up strategies for women treated for early breast cancer. Cochrane Db Syst Rev. 2005. 


\section{Synopsis:}

A national study of surveillance systemic imaging following locoregional breast cancer treatment in 1,217 Commission on Cancer-accredited facilities found patients continue to undergo asymptomatic systemic imaging in follow-up. Providers appear to tailor use based on cancer-related factors that confer increased recurrence risk. 


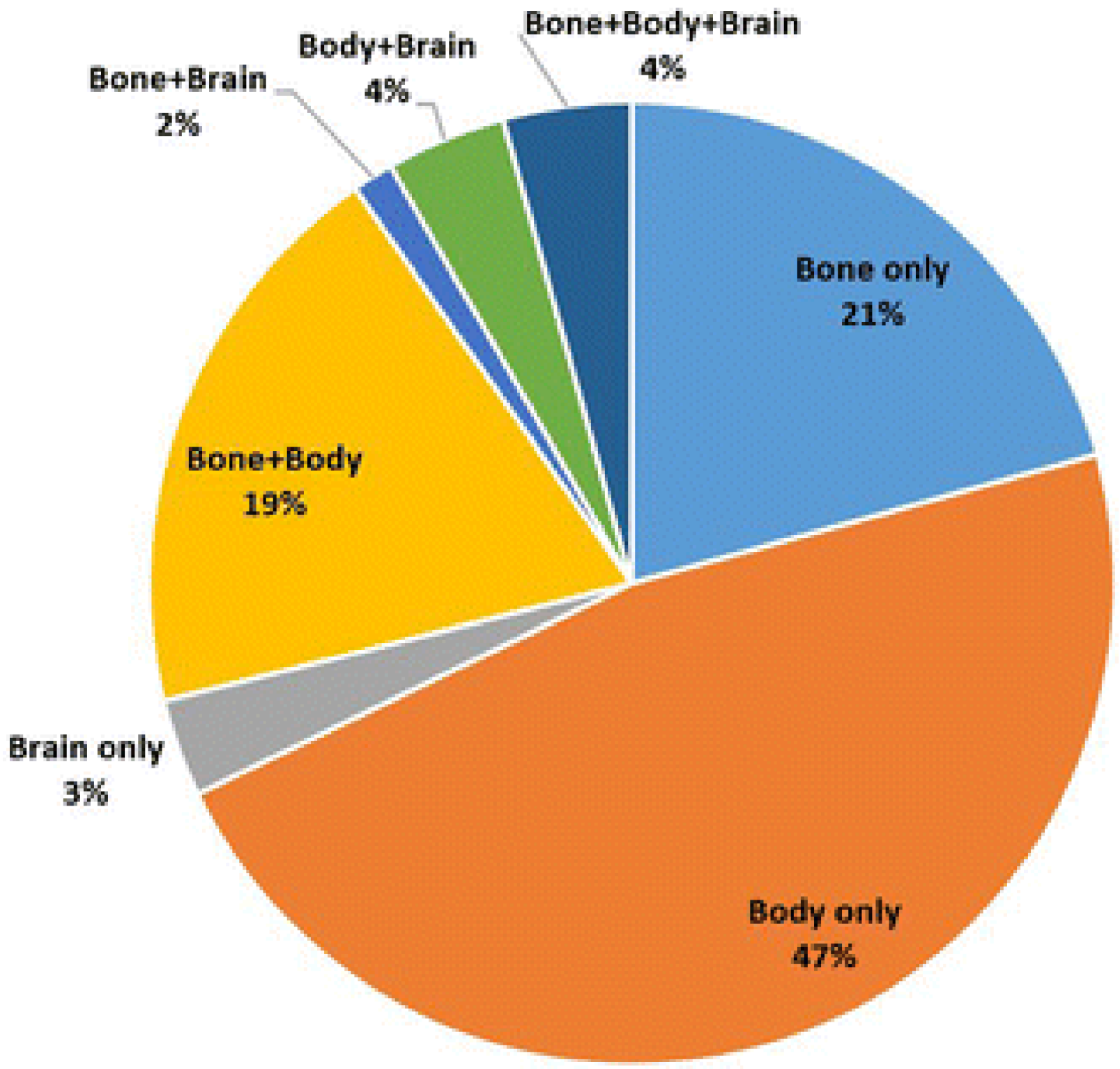

Figure 1.

Distribution of surveillance scan types for stage II-III breast cancer patients who received one or more surveillance scans during the follow-up period $(\mathrm{N}=3,254)$ 
Table 1.

Characteristics of stage II-III breast cancer patients at the time of diagnosis and first course treatment $(\mathrm{n}=10,838)^{\dagger}$

\begin{tabular}{|c|c|c|c|c|c|}
\hline Patient Characteristics & $\mathbf{N}$ & Percent & Patient Characteristics & $\mathbf{N}$ & Percent \\
\hline Sociodemographic Characteristics & & & Tumor Characteristics & & \\
\hline Age & & & Stage at Diagnosis & & \\
\hline$<50$ & 3041 & 28 & II & 7724 & 71 \\
\hline $50-69$ & 5126 & 47 & III & 3114 & 29 \\
\hline$\geq 70$ & 2671 & 25 & Grade & & \\
\hline Race & & & I & 1344 & 12 \\
\hline White & 9119 & 84 & II & 4381 & 40 \\
\hline Black & 1249 & 12 & III & 4494 & 41 \\
\hline Other & 470 & 4 & Unknown & 619 & 6 \\
\hline Hispanic Ethnicity & & & Histology & & \\
\hline No & 9207 & 85 & Ductal & 9055 & 84 \\
\hline Yes & 576 & 5 & Lobular & 1095 & 10 \\
\hline Unknown & 1055 & 10 & Other & 688 & 6 \\
\hline $\begin{array}{l}\text { Mean Percent in Zipcode with } \\
\text { Less than HS Degree (SD) }\end{array}$ & & & ER, PR, Her2 Risk Group & & \\
\hline $29 \%$ or more & 1780 & 16 & ER or PR Pos, Her2neu Neg & 6354 & 59 \\
\hline $20-28.9 \%$ & 2786 & 26 & ER and PR Neg, Her2neu Neg & 1647 & 15 \\
\hline $14 \%-19.9 \%$ & 3642 & 34 & ER or PR Pos, Her2neu Pos & 1318 & 12 \\
\hline Less than $14 \%$ & 2414 & 22 & ER and PR Neg, Her2neu Pos & 755 & 7 \\
\hline Unknown & 216 & 2 & Unknown ER/PR and/or Her2neu & 764 & 7 \\
\hline Insurance Status & & & Chemotherapy & & \\
\hline Private Insurance/Managed Care & 5903 & 54 & No & 2694 & 25 \\
\hline Medicaid & 818 & 8 & Yes & 7981 & 74 \\
\hline Medicare \& Other Government & 3650 & 34 & Unknown & 163 & 2 \\
\hline Uninsured/Self-Pay/Insiirance Unknown & 467 & 4 & Surgery \& Radiation Therapies & & \\
\hline Urban/Rural & & & Breast Conserving Surgery + Radiation & 4287 & 40 \\
\hline Urban & 10117 & 93 & Breast Conserving Surgery Only & 499 & 5 \\
\hline Rural & 243 & 2 & Mastectomy + Radiation & 2771 & 26 \\
\hline Missing & 478 & 4 & Mastectomy Only & 3064 & 28 \\
\hline Clinical Characteristics & & & Unknown Radiation & 217 & 2 \\
\hline Charlson/Deyo Score (SD) & & & Facility Type & & \\
\hline 0 & 9195 & 85 & Community Cancer Program/Other & 3121 & 29 \\
\hline \multirow[t]{2}{*}{$1+$} & 1643 & 15 & Comprehensive Community Cancer Program & 5775 & 53 \\
\hline & & & Academic/Research Program & 1942 & 18 \\
\hline
\end{tabular}


Table 2.

Percent of patients with advanced imaging by intent of scan $(n=10,838)$

\begin{tabular}{lcccc}
\hline & Overall & Head & Body & Bone \\
\hline 1+ Cancer-related scans & $48.2 \%$ & $16.9 \%$ & $41.1 \%$ & $23.0 \%$ \\
1+ Surveillance Scans & $30.0 \%$ & $3.9 \%$ & $22.4 \%$ & $13.7 \%$ \\
2+ Surveillance Scans & $12.1 \%$ & $0.8 \%$ & $9.5 \%$ & $4.3 \%$ \\
\hline
\end{tabular}


Table 3.

Percent of patients with stage II-III breast cancer within each follow-up year that received at least one surveillance advanced imaging scan $(\mathrm{N}=10,838)^{\dagger}$

\begin{tabular}{lccccc}
\hline ER, PR, HER2Neu Risk Group & $\mathbf{N}$ & $\begin{array}{c}\text { Year 1* } \\
(\mathbf{N = 1 0 , 8 3 8})\end{array}$ & $\begin{array}{c}\text { Year 2* } \\
(\mathbf{N = 1 0 , 1 1 4})\end{array}$ & $\begin{array}{c}\text { Year 3* } \\
(\mathbf{N = 9 , 5 1 7 )}\end{array}$ & $\begin{array}{c}\text { Year 4 } \\
(\mathbf{N = 9 , 0 6 1})\end{array}$ \\
\hline Overall & 10838 & $13.5 \%$ & $10.0 \%$ & $8.1 \%$ & $6.0 \%$ \\
ER or PR Pos, Her2neu Neg & 6354 & $\mathbf{1 2 . 4 \%}$ & $\mathbf{9 . 7 \%}$ & $\mathbf{7 . 9 \%}$ & $6.0 \%$ \\
ER and PR Neg, Her2neu Neg & 1647 & $\mathbf{1 6 . 4 \%}$ & $\mathbf{1 0 . 0 \%}$ & $\mathbf{7 . 9 \%}$ & $6.2 \%$ \\
ER or PR Pos, Her2neu Pos & 1318 & $\mathbf{1 4 . 6 \%}$ & $\mathbf{1 1 . 1 \%}$ & $\mathbf{9 . 9 \%}$ & $6.2 \%$ \\
ER and PR Neg, Her2neu Pos & 755 & $\mathbf{1 9 . 2 \%}$ & $\mathbf{1 4 . 6 \%}$ & $\mathbf{1 0 . 7 \%}$ & $7.2 \%$ \\
Unknown ER/PR and/or Her2neu & 764 & $\mathbf{8 . 8 \%}$ & $\mathbf{6 . 9 \%}$ & $\mathbf{4 . 1 \%}$ & $4.5 \%$ \\
\hline
\end{tabular}

${ }^{\dagger}$ Patients were removed from follow-up years the year after death or being diagnosed with a distant recurrence

* Bolded values indicate significant difference between ER/PR, Her2Neu Status Risk Group and Receipt of Imaging, p<0.001 
Table 4.

Descriptive statistics and adjusted association between patient and treatment factors associated withreceipt of one ormore surveillance advanced imaging studies during follow-up period among women with stage II-III breast cancer $(\mathrm{N}=10,838) \dagger$

\section{Overall}

Sociodemographic Characteristics

Age

$<50$

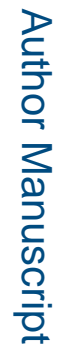

50-69

$\geq 70$

Race

White

Black

Other

Hispanic Ethnicity ${ }^{*}$

No

Yes

Percent in Zipcode with Less than High School Degree

$29 \%$ or more

20-28.9\%

$14 \%-19.9 \%$

Less than $14 \%$

Insurance Status

Private Insurance/Managed Care

Medicaid

Medicare \& Other Government

Uninsured/Self-Pay/Insurance Unknown

Urban/Rural *

Rural

Urban

Health and Tumor-Related Factors

Mean Charlson/Deyo Score (SD)

0

$1+$

AJCC Pathologic Stage

Stage II

Stage III

\begin{tabular}{|c|c|c|c|c|c|c|c|}
\hline \multirow{4}{*}{$\begin{array}{c}\mathbf{N} \\
10838\end{array}$} & \multicolumn{7}{|c|}{ One or More Surveillance Scans } \\
\hline & \multirow{3}{*}{$\begin{array}{c}\begin{array}{c}\text { Unadjusted } \\
\text { Proportion \% } \\
\text { Yes } \\
(\mathbf{N}=\mathbf{3 , 2 5 4}) \\
30\end{array} \\
\end{array}$} & \multicolumn{3}{|c|}{ Adjusted Odds Ratios } & \multicolumn{3}{|c|}{$\begin{array}{l}\text { Adjusted Average Predicted } \\
\text { Probability }\end{array}$} \\
\hline & & \multirow[t]{2}{*}{ OR } & \multicolumn{2}{|c|}{$95 \% \mathrm{CI}$} & $\%$ & \multicolumn{2}{|c|}{$95 \% \mathrm{CI}$} \\
\hline & & & & & $30 \%$ & $29 \%$ & $31 \%$ \\
\hline 3041 & 34 & REF & & & $31 \%$ & $29 \%$ & $33 \%$ \\
\hline 5126 & 31 & 0.96 & 0.86 & 1.06 & $30 \%$ & $29 \%$ & $31 \%$ \\
\hline 2671 & 23 & 0.88 & 0.75 & 1.04 & $28 \%$ & $26 \%$ & $31 \%$ \\
\hline 9119 & 30 & REF & & & $30 \%$ & $29 \%$ & $31 \%$ \\
\hline 1249 & 30 & 0.93 & 0.81 & 1.07 & $29 \%$ & $26 \%$ & $31 \%$ \\
\hline 470 & 30 & 0.89 & 0.72 & 1.10 & $28 \%$ & $24 \%$ & $32 \%$ \\
\hline 9207 & 30 & REF & & & $30 \%$ & $29 \%$ & $31 \%$ \\
\hline 576 & 33 & 1.04 & 0.86 & 1.26 & $31 \%$ & $27 \%$ & $34 \%$ \\
\hline 1780 & 32 & REF & & & $31 \%$ & $29 \%$ & $33 \%$ \\
\hline 2786 & 30 & 0.93 & 0.81 & 1.06 & $30 \%$ & $28 \%$ & $31 \%$ \\
\hline 3642 & 30 & 0.95 & 0.83 & 1.08 & $30 \%$ & $29 \%$ & $32 \%$ \\
\hline 2414 & 29 & 0.92 & 0.79 & 1.06 & $29 \%$ & $28 \%$ & $31 \%$ \\
\hline 5903 & 32 & REF & & & $30 \%$ & $29 \%$ & $31 \%$ \\
\hline 818 & 36 & 1.16 & 0.99 & 1.36 & $33 \%$ & $30 \%$ & $36 \%$ \\
\hline 3650 & 25 & 0.96 & 0.85 & 1.09 & $29 \%$ & $27 \%$ & $31 \%$ \\
\hline 467 & 35 & 1.14 & 0.93 & 1.40 & $33 \%$ & $28 \%$ & $37 \%$ \\
\hline 243 & 24 & REF & & & $22 \%$ & $17 \%$ & $27 \%$ \\
\hline 10117 & 30 & 1.51 & 1.12 & 2.04 & $30 \%$ & $29 \%$ & $31 \%$ \\
\hline 9195 & 30 & REF & & & $30 \%$ & $29 \%$ & $31 \%$ \\
\hline 1643 & 30 & 1.08 & 0.95 & 1.21 & $31 \%$ & $29 \%$ & $34 \%$ \\
\hline 7724 & 26 & REF & & & $28 \%$ & $27 \%$ & $29 \%$ \\
\hline 3114 & 39 & 1.46 & 1.32 & 1.62 & $35 \%$ & $34 \%$ & $37 \%$ \\
\hline
\end{tabular}




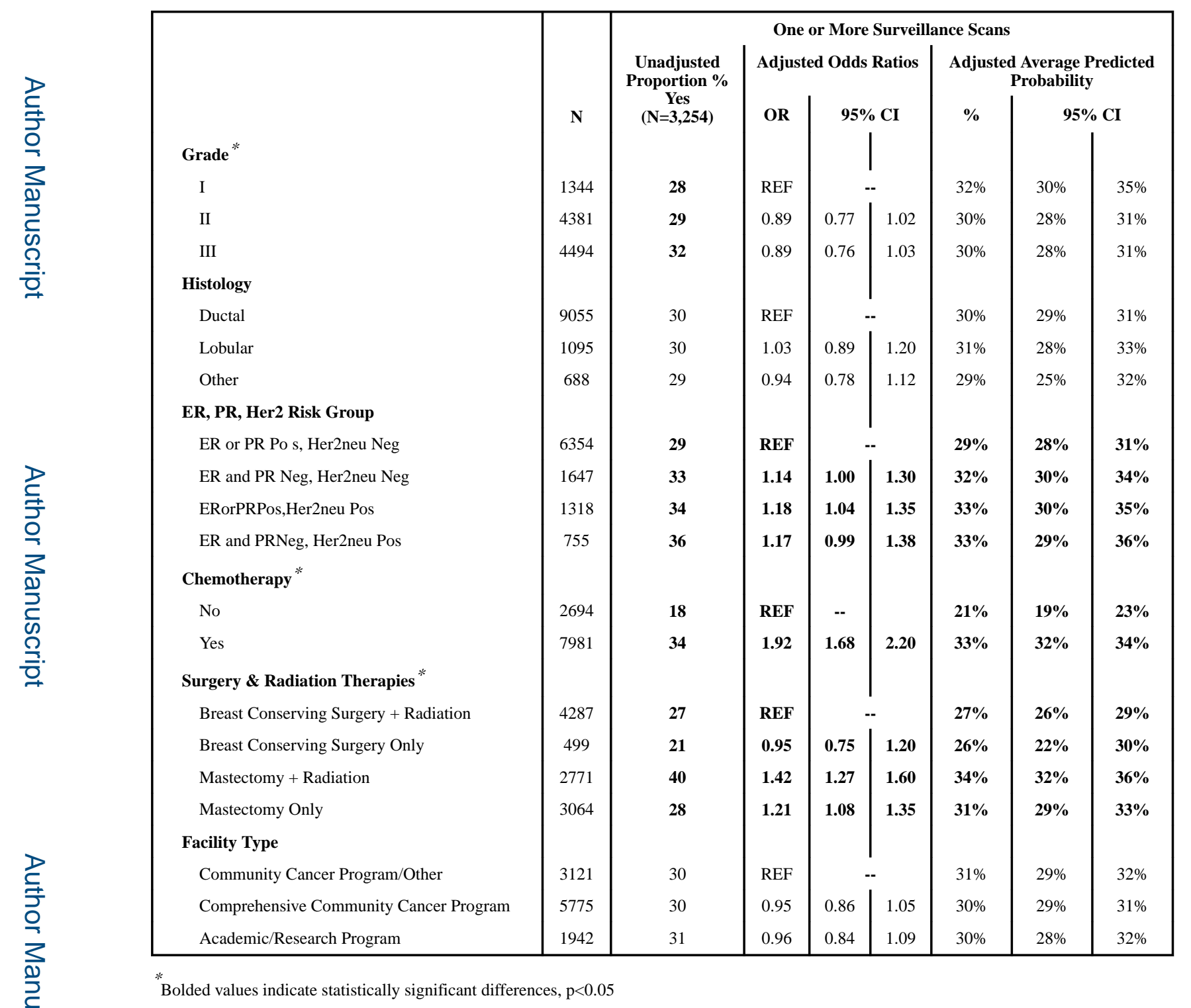

